\title{
Prediction of velocity distribution of laminar circular jet with an EMMS model
}

\author{
Kezhi Yü ${ }^{\mathrm{a}}$, Zhaoyang $\mathrm{Cao}^{\mathrm{b}}$, Yanling Liu ${ }^{\mathrm{c}}$ \\ Department of Refrigeration Engineering, Shanghai Ocean University, Shanghai 201306, China. \\ akzyu@shou.edu.cn, b 1349828737@qq.com, cylliu@shou.edu.cn
}

Keywords: energy-minimization multi-scale, circular jet, laminar flow, dissipation, Gauss distribution.

\begin{abstract}
The energy minimization multi-scale model is applied to the laminar circular jet. The shear dissipation tends to minimum, which can be used as the stability condition of laminar circular jet. The stability condition is adopted to predict the velocity distribution of laminar circular jet. Studies showed that the velocity distribution index of the laminar circular jet is 2.1 , which is difference from the traditional Gaussian distribution.
\end{abstract}

\section{Introduction}

EMMS (Energy-minimization Multi-scale ) model is a mechanism model, which is widely used in gas-solid two-phase flow, and has achieved great success [1-13]. Li Jinghai eatl. found that the coordination mechanism between the inertial and viscous effect in single-phase turbulence is very similar to that in a gas-solid system. Therefore, the EMMS model can be extended to turbulence field and the turbulence stability conditions is proposed. In this model, the shear viscous dissipation rate tends toward minimum and the total dissipation tends to maximum at the same time. This model predicted the turbulent pipe flow velocity distribution successfully [14, 15].

Jet flow is a common flow besides pipe flow. However, the EMMS model has not yet been applied to the study of jet flow. In this paper, we try to extend the energy minimum scale model to the research of circular jet. By using the stability condition of the circular jet, the velocity distribution of laminar and turbulent circular jet is predicted.

\section{Assumed velocity distribution in a circular jet}

In the fully developed region of the circular jet flow in the main section, the velocity distribution of each section is similar [16].

$$
\frac{u}{u_{m}}=f\left(\frac{r}{b_{e}}\right)
$$

where $b_{e}$ is the characteristic half thickness of the jet cross section, which is shown in Fig.1.

Based on the experimental results and the random nature of the turbulence, the assumption of reasonable velocity distribution:

$$
u=u_{m} e^{-\frac{r^{n}}{b_{e}^{n}}}
$$

where $n$ is a variable to be determined.

\section{The basic characteristics of circular jet}

Kinetic energy of arbitrary cross section. Kinetic energy

$$
\begin{aligned}
& E=\int_{0}^{\infty} 2 \pi r \rho u \cdot \frac{u^{2}}{2} d r=\rho \pi b_{e}^{2} u_{m}^{3} \int_{0}^{\infty} \eta e^{-3 \eta^{n}} d \eta=\rho \pi b_{e}^{2} u_{m}^{3} e(n) \\
& \text { where } e(n)=\int_{0}^{\infty} e^{-3 \eta^{n}} \eta d \eta \text {, is a function of the variable } n \text {. }
\end{aligned}
$$




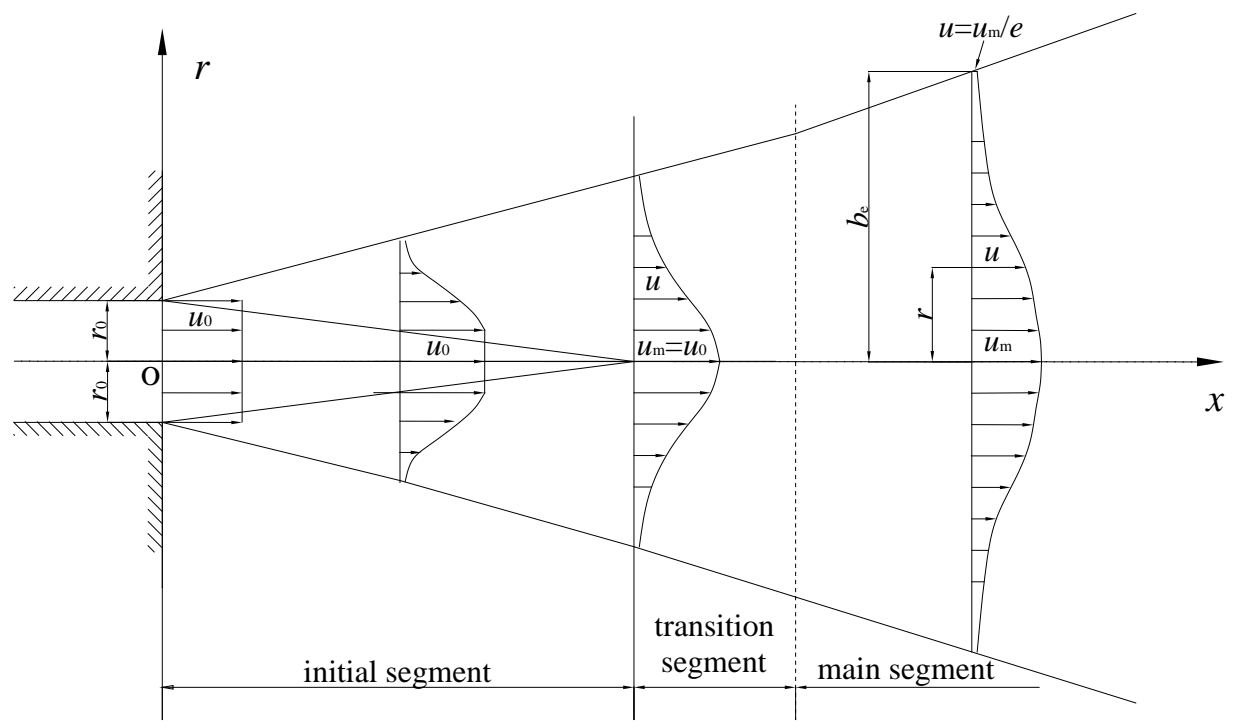

Fig.1.Main section of circular jet

The change of kinetic energy along the flow direction

$\frac{d E}{d x}=\rho \pi e(n) \frac{d\left(b_{e}^{2} u_{m}{ }^{3}\right)}{d x}$

Momentum of arbitrary cross section. Jet momentum

$M=2 \pi \int_{0}^{\infty} \rho r u^{2} d r=2 \rho \pi b_{e}^{2} u_{m}^{2} \int_{0}^{\infty} \eta e^{-2 \eta^{n}} d \eta=2 \rho \pi b_{e}^{2} u_{m}{ }^{2} m(n)$

where $m(n)=\int_{0}^{\infty} e^{-2 \eta^{n}} \eta d \eta$, is a function of the variable $n$.

The momentum of each section along the jet is equal to the momentum of the exit $M_{0}=\frac{\pi}{4} \rho D^{2} u_{0}^{2}$ where $D$ is the diameter of circular jet and $u_{0}$ is the jet exit velocity.

Therefore

$8 b_{e}^{2} u_{m}^{2} m(n)=D^{2} u_{0}^{2}$

Flux of arbitrary cross section. Jet flux

$Q=\int_{0}^{\infty} 2 \pi r \rho u d r=2 \rho \pi b_{e}^{2} u_{m} \int_{0}^{\infty} \eta e^{-\eta^{2}} d \eta=2 \rho \pi b_{e}^{2} u_{m} q(n)$

where $q(n)=\int_{0}^{\infty} e^{-\eta^{n}} \eta d \eta$, is a function of the variable $n$.

The change of flux along the flow direction

$\frac{d Q}{d x}=-2 \rho \pi q(n) b_{e}^{2} \frac{d u_{m}}{d x}$

It can be expressed as

$d Q=2 d x \rho \pi b_{e} \alpha u_{m}$

where $\alpha$ is entrainment factor.

$\alpha=\frac{-2 \rho \pi q(n) b_{e}^{2} \frac{d u_{m}}{d x}}{2 \rho \pi b_{e} u_{m}}=-q(n) \frac{b_{e}}{u_{m}} \frac{d u_{m}}{d x}$

The relationship among parameters. For a circular jet [16], 
$\frac{u_{m}}{u_{0}}=a \frac{D}{x}$

$b_{e}=\varepsilon x$

where $a$ and $\varepsilon$ are constants.

According to the Equation (7), the relationship between these constants is

$a \varepsilon=\frac{1}{\sqrt{8 m(n)}}$

According to the Equation (10), entrainment factor can be expressed as:

$\alpha=q(n) \varepsilon$

\section{Stability analysis of laminar circular jet flow}

In a circular jet, the local shear dissipation of unit volume can be expressed as [17]

$$
W_{v}(y)=\mu\left(\frac{d u}{d y}\right)^{2}
$$

Its integral over the whole jet section

$\bar{W}_{v}=2 \pi \int_{0}^{\infty} r W_{v} d r=2 \pi n^{2} \mu u_{m}^{2} \int_{0}^{\infty} \eta^{2 n-1} e^{-2 \eta^{n}} d \eta=\frac{n \pi \mu u_{m}{ }^{2}}{2}$

According to the stability condition of the laminar flow [18]

$\overline{W_{v}} \rightarrow \min$

From Equations (11), (13), (14) and (16), Equation (17) can be expressed as:

$\frac{\pi \mu D^{2} u_{0}^{2}}{16 x^{2} \alpha^{2}} \frac{n q^{2}(n)}{m(n)} \rightarrow \min$

If $\alpha$ is a constant, the stability condition of laminar circular jet can be expressed as:

$W(n)=\frac{n q^{2}(n)}{m(n)} \rightarrow \min$

\section{Results analysis and discussion}

In order to obtain the velocity distribution of laminar circular jet, the Equation (19) can be used as the optimizing condition. According to Equation (19), the value of $W$ is dependent on the value of $n$, which is shown in Fig.2.

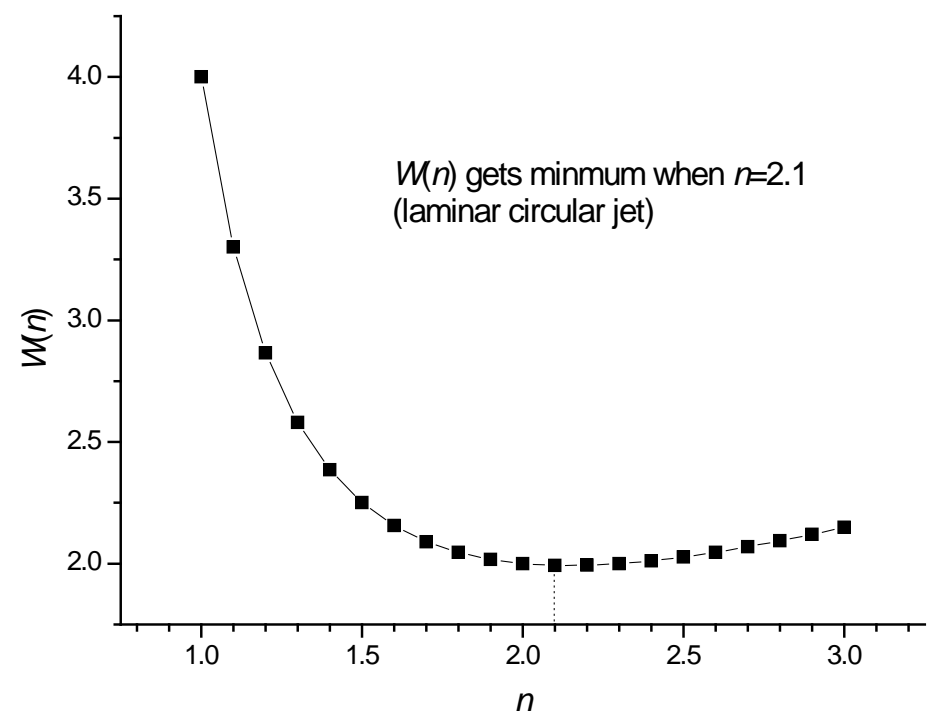

Fig.2. The relationship between of $W(n)$ and $n$ in laminar circular jet 
From Fig.2, with the increment of $n, W(n)$ first decreases and then increases. When $n$ equal 2.1, $W(n)$ obtains minimum. It is obvious that $n=2$ will lead to recognized Gaussian distribution. Therefore, this result is different from Gaussian distribution [16, 19].

$\mathrm{Xu}$ eatl. found that the velocity distribution is different from the Gaussian normal distribution when the Reynolds number is small through the experiment. In order to analyze the results of the EMMS model and the Gauss distribution, we can make the corresponding velocity distribution curve, as shown in Fig.3. From the Fig.3, the EMMS model shows better agreement with the experimental results (Fig. 4 in Ref [19] )than Gauss distribution.

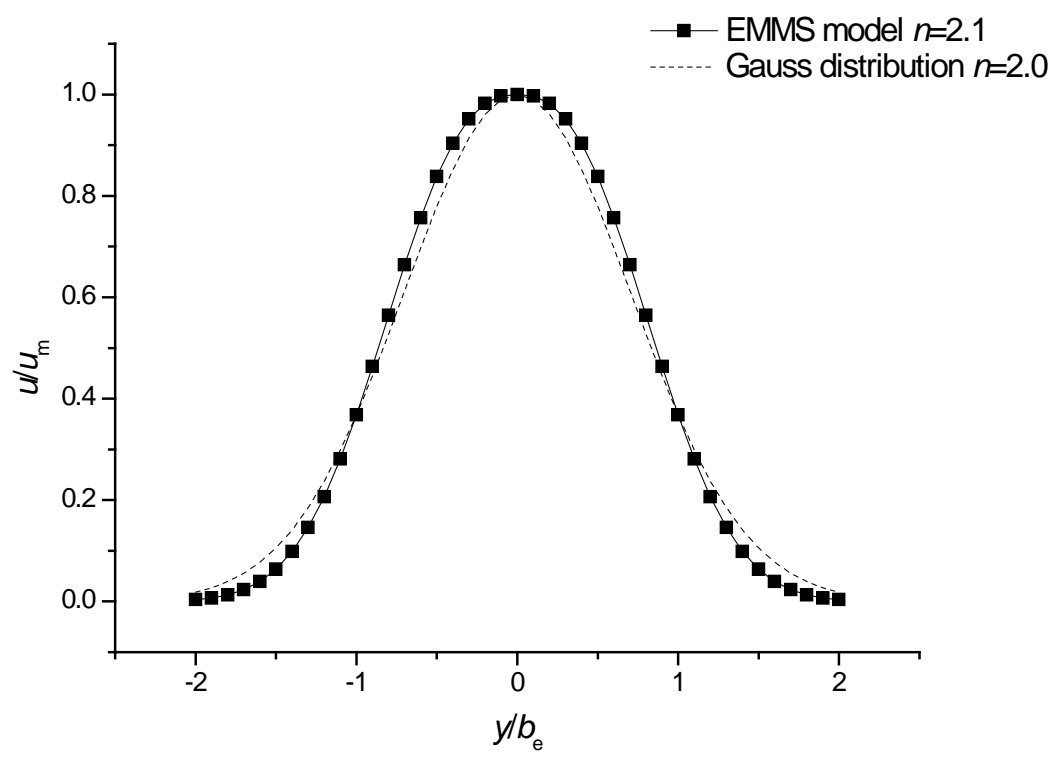

Fig.3. Comparison of velocity distribution by an EMMS model and Gauss distribution in laminar circular jet

\section{Summary}

In this paper, the EMMS model is applied to the research of circular jet. The stability condition is used to predict the velocity distribution of the laminar circular jet. The results show that the velocity distribution index of the laminar circular jet is 2.1. The conclusion is different from the traditional Gauss distribution function, but is more consistent with the experimental results.

\section{Acknowledgment}

This work was financially supported by Science and technology support program of Shanghai Science and Technology Commission (13dz1203002)

\section{References}

[1] Cheng Congli, Gao Shiqiu , Zhang Zhongdong. Energy-minimization multi-scale core-annulus (EMMS/CA) model for gas-solid circulating fluidized beds [J]. CIESC Journal,2002,53(8): 804-809.

[2] LiJingha, MoosonKwauk. Introduction to EMMS model for fluid particle two phase flow[J]. Chemical Engineering, 1992, 20(2):26-29.

[3] Chen Sheng, Wang Wei, Yan Zihan, Lu Chunxi, Fan Yiping. Simulation of gas-solid mixing and flow in the feed stock zone of a riser by EMMS drag model[J]. Chemical Reaction Engineering and Technology,2014,30(1):71-78.

[4] Chen Cheng, Qi Haiyin. Development and validation of cluster and EMMS drag model [J]. CIESC Journal,2014,65(6):2003-2012. 
[5] LI Fei, Chen Cheng,Wang Jinsheng,Qi HaiYing. QL-EMMS drag model \& its revision for flu1dized dense gas-solid two-phase flow [J]. Journal of Engineering Thermophicics, 2011, 32(1):75-79.

[6] Yun Zhang, Wei Ge, Xiaowei Wang, Chaohe Yang. Validation of EMMS-based drag model using lattice Boltzmann simulations on GPUs[J]. Particuology,2011,04:365-373.

[7] Huo Men,Zhang Nan,,Meng Fanyong,Wang we,Huang weixin. EMMS-based 3D full-loop CFD simulation of a circulating fluidized bed [J]. Computer sand Applied Chemistry,2013,30(3):223-228.

[8] Cheng Changjian,Ge Wei. Further analysis on the choking criteria in the EMMS model [J]. Progress In Chemistry,2008,20(4):620-624.

[9] Lin Zhang, Xiaoping Qiu,Limin Wang,Jinghai Li. A stability condition for turbulence model: From EMMS model to EMMS-based turbulence model [J]. Particuology, 2014,05:142-154.

[10]Bona Lu,Nan Zhang,Wei Wang, Jinghai Li. Extending EMMS-based models to CFB boiler applications[J]. Particuology [J], 2012, 06:663-671.

[11]Junwu Wang. Flow structures inside a large-scale turbulent fluidized bed of FCC particles: Eulerian simulation with an EMMS-based sub-grid scale model[J]. Particuology, 2010, 02:176-185.

[12]Bona Lu, Nan Zhang, Wei Wang, Jinghai Li. Extending EMMS-based models to CFB boiler applications [J]. Particuology, 2012, 10,663-671.

[13]Ning Yang,Wei Wang,Wei Ge, Jinghai Li. Modeling of meso-scale structures in particle-fluid systems: the EMMS/CFD approach[J]. China Particuology Science and Technology of Particles, 2005,3(1-2):78-79.

[14]Jinghai Li., Zhongdong Zhang, Wei Ge, Qicheng Sun, Jie Yuan. A simple varational criterion for turbulent flow in pipe [J]. Chemical Engineering Science,1999,54: 1151-1154.

[15]Li Jinghai, Zhang Zhongdong, Ge Wei, Yuan Jie. Extreme conditions for two mechanisms coexistence of dissipative structures [J]. Chinese Science Bulletin, 1999, 44(6): 613-617.

[16]Yu Changzhao. Turbulent jet[M] (Beijing: High Education Press, 1993

[17]Bejan A. Entropy Generation through Heat and Fluid Flow [M]. New York: Wiley, 1982

[18]Wang L M. Discrete simulation for single-phase complex flows [D] Beijing: Chinese Academy of Sciences), 2008.

[19]Xu Shikai, Wang Yong. Determination of critical Reynolds number at outlets of free jet flow [J]. Journal of Hehai University(Natural science edition),2007,35(6): 699-704. 